\title{
MR Arthrography of Shoulder
}

The glenohumeral joint boasts the greatest range of motion of any peripheral joint in the body, but not without cost; it is also the most frequently dislocated joint in the body. Stability of this articulation is limited for two major reasons. The articulating surface of the glenoid is significantly smaller than that of the humeral head, and the joint capsule is redundant and provides little support.

Generally speaking, the term instability when applied to the glenohumeral joint, refers to a recurrence of dislocation or subluxation. This is not an unexpected complication, given the complex biomechanics of this articulation, the sophisticated movement achieved, and the high performance demanded from the body. There is much debate and controversy surrounding the mechanism and imaging evaluation of shoulder instability.

This unit will focus on an MR arthrography protocol for evaluation of glenohumeral joint instability.

\section{ROLE OF EVALUATION IN SHOULDER INSTABILITY}

As previously mentioned, MR imaging has revolutionized the evaluation of the glenohumeral joint, in particular, internal derangements. It offers excellent visualization of soft tissue structures and unparalleled soft tissue contrast. Although conventional MR imaging allows direct visualization of the major anatomic structures of the glenohumeral joint, many investigators feel smaller intraarticular structures and their pathology are difficult to evaluate without distention of the joint capsule (Flanagan et al., 1990; Chandnani et al., 1993; Chung et al., in press). The disadvantages of MR arthrography include the necessity for a more complex scheduling system as the arthrography portion of the study is usually performed fluoroscopically, and the transformation of a noninvasive study to an invasive one. Though this remains a topic of debate, the authors feel MR arthrography is the imaging method of choice for evaluation of glenohumeral joint instability and will continue to gain acceptance in the community as more sophisticated study interpretation is demanded from referring physicians.

This unit will present an MR arthrography protocol for evaluation of glenohumeral joint instability. It will not cover the arthrogram technique as this is performed as a completely separate procedure. It will not cover the arthrogram technique as this is performed as a completely separate procedure.

Although the arthrogram technique will not be discussed in detail, the operator has the choice of media with which to distend the glenohumeral joint. In the early literature, it was felt that dilute gadolinium solutions offered optimal contrast for delineation of intraarticular structures as compared to several other agents including saline (Hajek et al., 1987). Although this has been validated in the recent literature where superior contrastto-noise ratios have been demonstrated with dilute gadolinium ( $4 \mathrm{mmol} / \mathrm{l}$ gadoteridate) MR arthrography as compared with saline MR arthrography, the differences in diagnostic efficacy have not proven significant (Zanetti et al., 1997). The primary technical differences resulting from the use of saline as the contrast medium would be the emphasis on fluid sensitive sequences such as proton density fat-suppressed fast spin echo (FSE), $T_{2}$-weighted fat-suppressed fast spin echo, or inversion recovery imaging to optimally evaluate the distribution of saline in the joint. The authors feel the advantages of using a dilute gadolinium solution as the contrast agent in conjunction with $T_{1}$-and $T_{2}$-weighted images offer the significant advantage of distinguishing between existing native fluid collections and those resulting from the intraarticular distention. Table A22.2.1 lists

Contributed by Christine B. Chung, Leopoldo M. Gigena, and Donald Resnick

Current Protocols in Magnetic Resonance Imaging (2001) A22.2.1-A22.2.13

Copyright $\odot 2001$ by John Wiley \& Sons, Inc.
BASIC

PROTOCOL

Shoulder

A22.2.1 


\begin{tabular}{ll}
\hline Coil type & Shoulder phased array or standard shoulder coil \\
Gradient coil strength & $22 \mathrm{mT} / \mathrm{m}$ \\
Cardiac gating & No \\
Peripheral gating & No \\
Respiratory gating & No \\
Oxygen & No \\
Motion cushions & Useful \\
Use of contrast agents & Yes (intraarticular) \\
\hline
\end{tabular}

hardware and parameters needed to perform the examination. This protocol will take $\sim 45$ min to perform.

\section{Set up patient and equipment}

1. The patient will be scheduled for a two-part examination. The first part is the standard arthrogram (where contrast is administered into the articulation), which is scheduled 30 min prior to the MR imaging portion of the study. The coordination of the timing of these two components is of the utmost importance. After appropriate informed consent has been obtained and the arthrogram is completed, transport the patient to the MR imaging suite with minimal movement of the shoulder being imaged to decrease the possibility of rupturing the joint capsule. It is best to have them transported by wheelchair.

2. Begin MR imaging within 15 to 30 min of the arthrogram procedure to lessen the possibility of resorption of the contrast material.

3. Screen the patient by having him/her complete a standardized form designed to ensure that he or she has no internal ferromagnetic materials. For questions regarding safe scanning of implants, see Shellock (1996). In the authors' screening process, specific reference is made to the following items, which can prove to be a health hazard to the patient or interfere with image acquisition. These include:
a. Cardiac pacemaker
b. Retained metal fragments in eyes
c. Heart valve replacement, venous umbrella
d. Vascular clips
e. Prosthetic devices in the eyes and joints
f. Hearing aid, neurostimulator, insulin pump
g. Intrauterine device (I.U.D.; birth control device)
h. Shunts/stents (ventricular, spinal, biliary), metal mesh/coil implant
i. Orthopedic hardware

4. In addition, the screening form includes general inquiries to health issues that are pertinent to the performance of the MR imaging study, the need for any emergency equipment (e.g., crash cart, oxygen), and its interpretation. These include:

a. Pregnancy status of patient

b. Respiratory difficulties or nausea when lying in supine position

c. Past medical history and current medications

d. History of claustrophobia 
5. In conjunction with the standardized screening form, have the patient complete a background information form.

This proves to be an invaluable source of history and clinical information given the paucity of information often provided on the requisition forms. The information that the patient provides may help the technician to do a more specific study and the radiologist to render the most accurate interpretation of the exam.

The questionnaire includes:

a. Requesting physician and subspecialty

b. Origin and progression of symptoms

c. Request for diagram of the symptomatic area

d. Activities that exacerbate symptoms

6. Review the data sheets and interview the patient to ensure he or she understands the nature of all questions and that the data sheets have been filled out as completely and accurately as possible.

7. Have the patient sign any necessary consent forms.

8. Instruct the patient to remove all jewelry or metal from their body, and change into a gown.

9. Explain what will happen during the course of the procedure and what the patient will experience while in the magnet.

a. The patient will be given earphones to wear during the examination to protect from the loud knocking sound produced during the study. This knocking will occur when images are being acquired, take place six to seven times and last a few minutes each time. The technologist and patient may communicate at any time during the study by simply speaking out, though it is preferable to wait until the knocking sound has stopped.

b. The patient is asked to remain quiet and avoid any movement during the time images are being acquired-i.e., when the knocking sound is occurring.

c. The patient will be provided with a safety squeeze-bulb. Demonstrate how it works and explain to the patient when to use the squeeze-bulb (i.e., if they need assistance during the exam).

10. Have the patient mount onto the table and assume a supine position. Place a wedge under the patient's knees to facilitate their comfort and lessen the likelihood of motion. Cover the patient with a sheet to maintain personal privacy.

11. The arm should be fully extended at the patient's side and in neutral position. This is achieved by having the thumb pointed towards the ceiling (see Fig. A22.2.1). When the thumb points away from the body, the humerus is in external rotation, when it points towards the body, it is in internal rotation.

12. Place a dedicated shoulder coil over the region of the glenohumeral joint (see Fig. A22.2.1).

13. The centering light is used to assign a reference point of the patient's position on the table relative to the bore of the magnet and localize the region of the shoulder to be imaged. Then place the patient into the bore of the magnet. In order to preserve a true frame of reference from one scan relative to another, the patient must not move after this point. 
Sequence 1: Spoiled gradient-recalled (SPGR) coronal localizer (pilot scan)

14. This large field of view localizer will result in an image of the upper extremity, axilla, and thorax. From this, the transverse images are planned from the beginning of the soft tissues just above the $\mathrm{AC}$ joint to a point below the inferior most aspect of the glenoid (see Fig. A22.2.2). Let patient know that you are ready. Run sequence 1 according to the parameters in Table A22.2.2.

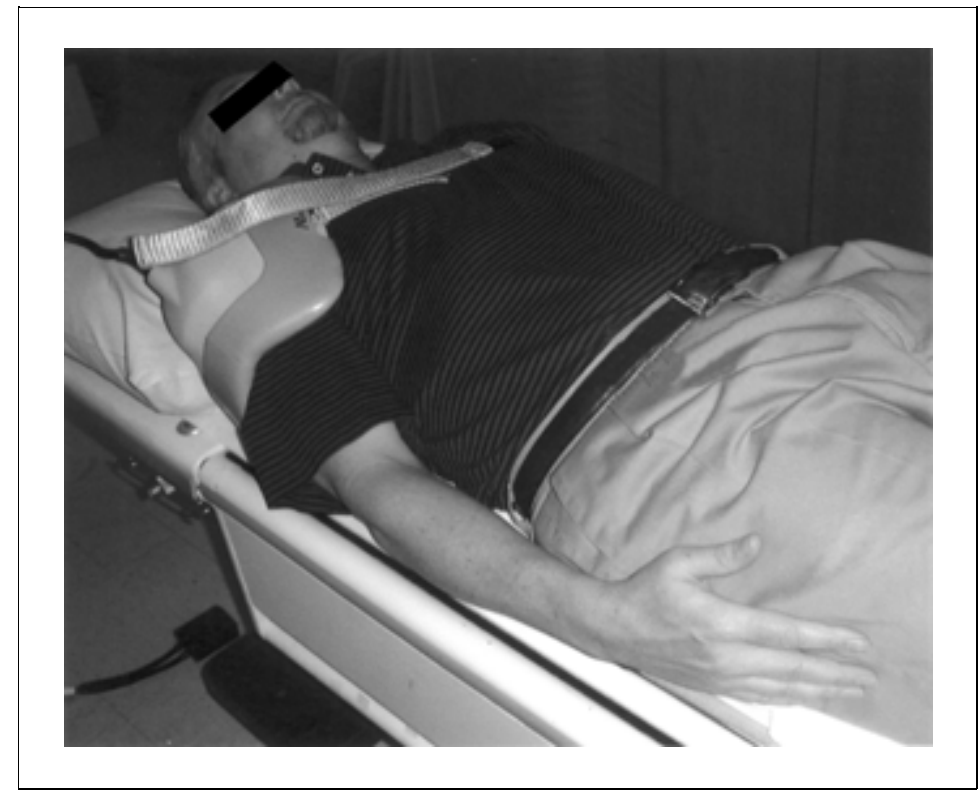

Figure A22.2.1 The arm is extended at the patient's side and in neutral position, with the thumb pointed towards the ceiling. The coil is placed over the glenohumeral joint.

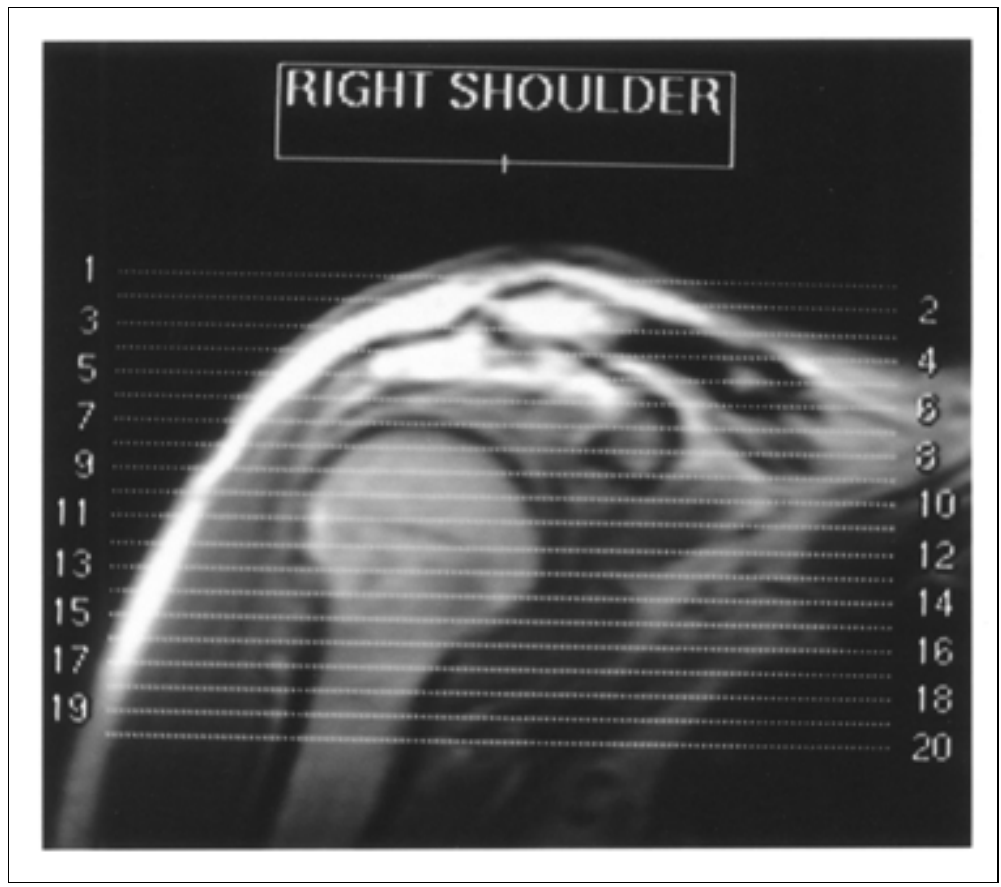

Figure A22.2.2 SPGR coronal localizer. The grid lines are set from above the acromioclavicular joint and extend to a point below the inferior aspect of the glenohumeral joint. 
Table A22.2.2 Clinical Parameters for Sequence 1: Spoiled Gradient-Recalled (SPGR) Coronal Localizer (Pilot Scan)

\begin{tabular}{ll} 
Patient position & Supine \\
Scan type & 2-D spoiled gradient-recalled \\
Imaging plane (orientation) & Coronal localizer \\
Central slice or volume center & Center of anatomy \\
Echo time $\left(T_{\mathrm{E}}\right)$ & $4.2 \mathrm{msec}$ \\
Repeat time $\left(T_{\mathrm{R}}\right)$ & $150 \mathrm{msec}$ \\
Flip angle $(\mathrm{FA})$ & $60^{\circ}$ \\
Fields of view $(\mathrm{FOV}, \mathrm{FOV}$ & $\mathrm{F})$ \\
Resolution $(\Delta x, \Delta y)$ & $360 \mathrm{~mm}, 360 \mathrm{~mm}$ \\
Number of data points collected $\left(N_{\mathrm{x}}, N_{\mathrm{y}}\right)$ & $1.41 \mathrm{~mm}, 2.82 \mathrm{~mm}$ \\
Display matrix $\left(D_{\mathrm{x}}, D_{\mathrm{y}}\right)$ & 256,128 \\
Slice thickness $(\Delta z)$ & 512,512 \\
Number of slices & $7 \mathrm{~mm}$ \\
Slice gap & 13 \\
Number of acquisitions $\left(N_{\mathrm{acq}}\right)$ & $2 \mathrm{~mm}$ \\
Swap read and phase encoding & 2 \\
Saturation pulses & No \\
Scan time & None \\
\hline
\end{tabular}

Table A22.2.3 Clinical Parameters for Sequence 2: Transverse Fat-Suppressed Fast Spin Echo (FSE) $T_{1}$-Weighted

Patient position

Scan type

Imaging plane (orientation)

Pulse sequence database (PSD)

Central slice or volume center

Echo time $\left(T_{\mathrm{E}}\right)$

Receiver bandwidth (RBW)

Echo train length (ETL)

Repeat time $\left(T_{\mathrm{R}}\right)$

Flip Angle (FA)

Fields of view $\left(\mathrm{FOV}_{\mathrm{x}}, \mathrm{FOV}_{\mathrm{y}}\right)$

Resolution $(\Delta x, \Delta y)$

Number of data points collected $\left(N_{\mathrm{x}}, N_{\mathrm{y}}\right)$

Slice thickness $(\Delta z)$

Number of slices

Slice gap

Number of acquisitions $\left(N_{\text {acq }}\right)$

Swap read and phase encoding

Saturation pulses

Fat suppression

Scan time
Supine

Fast spin echo

Transverse

FSE-XLC

Acromioclavicular joint to inferior glenoid

$12.2 \mathrm{msec}$

$17.86 \mathrm{KHz}$

2

$650 \mathrm{msec}$

$90^{\circ}$

$160 \mathrm{~mm}, 160 \mathrm{~mm}$

$0.63 \mathrm{~mm}, 0.83 \mathrm{~mm}$

256, 192

$3 \mathrm{~mm}$

27

$1 \mathrm{~mm}$

2

No

Inferior

Yes

$6 \mathrm{~min}, 23 \mathrm{sec}$ 
Sequence 2: Transverse fat-suppressed fast spin echo (FSE) $T_{1}$-weighted

15. After the grid lines (graphic prescriptions) are set, changes in the sequence prescription can still be made. Check to see that all parameters have been correctly entered according to Table A22.2.3 before running this sequence.

16. Let the patient know that you will begin the scan and run it.

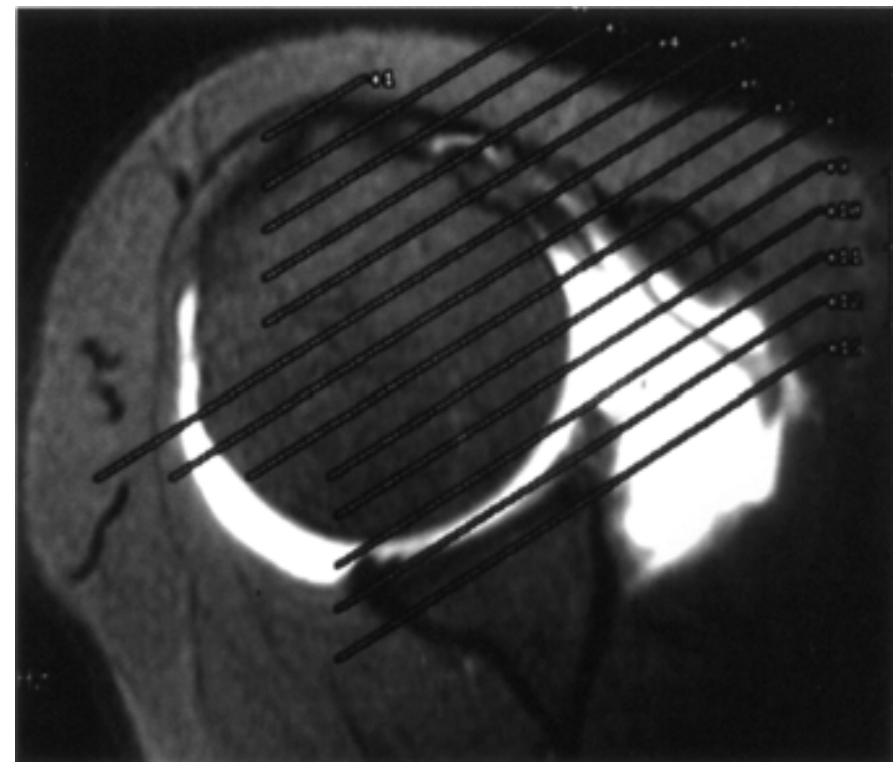

Figure A22.2.3 The grid lines are placed perpendicular to the spine of the scapula to perform the sagittal oblique images.

Table A22.2.4 Clinical Parameters for Sequence 3: Sagittal Oblique Fat-Suppressed Fast Spin Echo (FSE) $T_{1}$-Weighted

Patient position

Scan type

Imaging plane (orientation)

Pulse sequence database (PSD)

Central slice or volume center

Echo time $\left(T_{\mathrm{E}}\right)$

Receiver bandwidth (RBW)

Echo train length (ETL)

Repeat time $\left(T_{\mathrm{R}}\right)$

Flip angle (FA)

Fields of view $\left(\mathrm{FOV}_{\mathrm{x}}, \mathrm{FOV}_{\mathrm{y}}\right)$

Resolution $(\Delta x, \Delta y)$

Number of data points collected $\left(N_{\mathrm{x}}, N_{\mathrm{y}}\right)$

Slice thickness $(\Delta z)$

Number of slices

Slice gap

Number of acquisitions $\left(N_{\text {acq }}\right)$

Swap read and phase encoding

Saturation pulses

Fat suppression

Scan time
Supine

Fast spin echo

Sagittal oblique

FSE-XLC

Perpendicular to the long axis of the spine of the scapula

$12.4 \mathrm{msec}$

$17.86 \mathrm{KHz}$

2

$550 \mathrm{msec}$

$90^{\circ}$

$140 \mathrm{~mm}, 140 \mathrm{~mm}$

$0.55 \mathrm{~mm}, 0.73 \mathrm{~mm}$

256, 192

$3 \mathrm{~mm}$

24

$1 \mathrm{~mm}$

2

No

Inferior

Yes

$5 \mathrm{~min}, 24 \mathrm{sec}$ 
17. After the images have been acquired, if they are satisfactory in appearance, both the sagittal and coronal oblique imaging planes can be planned from the transverse images.

\section{Sequence 3: Sagittal oblique fat-suppressed fast spin echo (FSE) $T_{1}$-weighted}

18. Review the transverse images and select an image at the level of the mid-scapula in which the scapular spine is visualized. Place the localizer line parallel to the articular surface of the glenoid and perpendicular to the spine of the scapula (see Fig. A22.2.3).

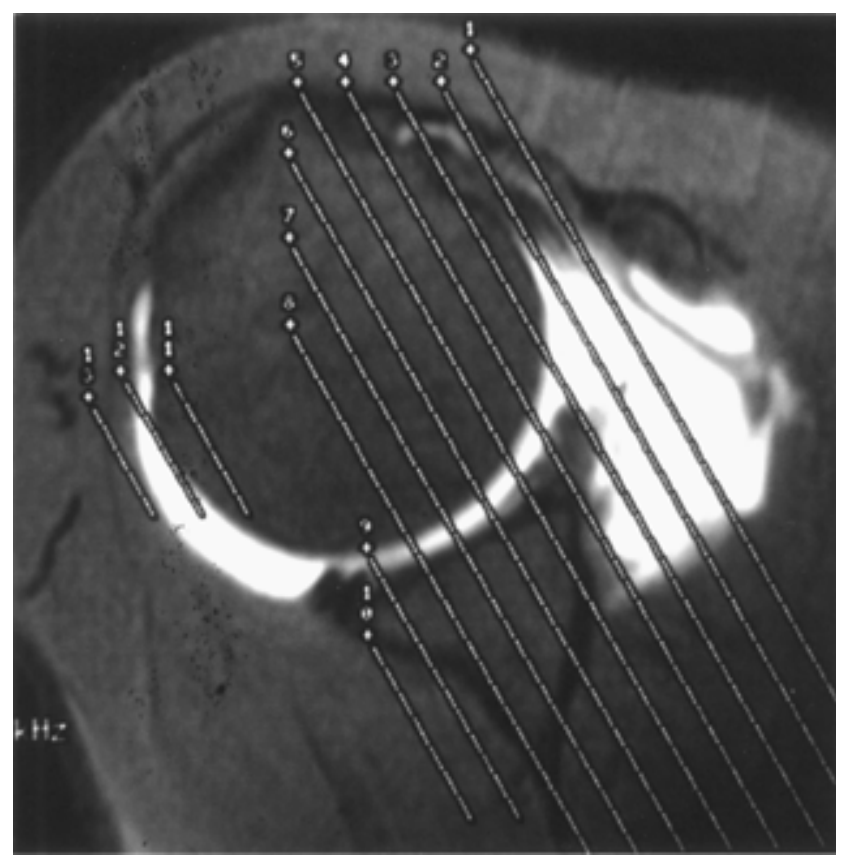

Figure A22.2.4 The grid lines are placed parallel to the spine of the scapula to perform the coronal oblique images.

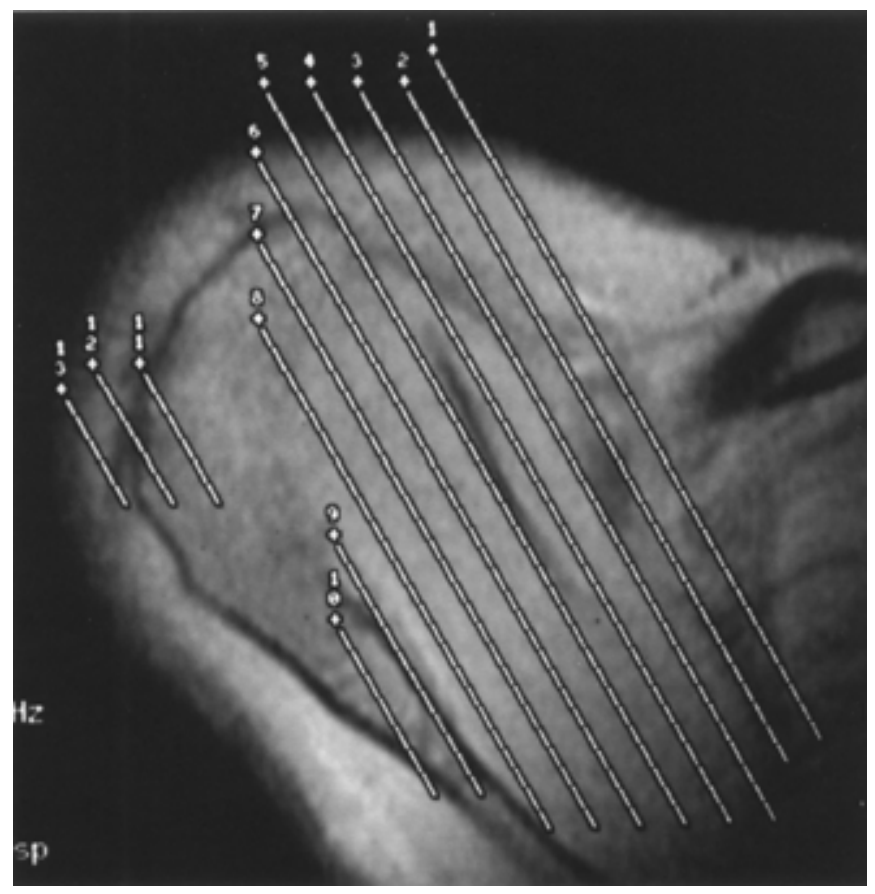

Figure A22.2.5 The grid lines are located parallel to the supraspinatus tendon to perform the coronal oblique images. 
Table A22.2.5 Clinical Parameters for Sequence 4: Coronal Oblique Fat-Suppressed Fast Spin Echo (FSE) $T_{1}$-Weighted

Patient position

Scan type

Imaging plane (orientation)

Pulse sequence database (PSD)

Central slice or volume center

Echo time $\left(T_{\mathrm{E}}\right)$

Receiver bandwidth (RBW)

Echo train length (ETL)

Repeat time $\left(T_{\mathrm{R}}\right)$

Flip angle (FA)

Fields of view $\left(\mathrm{FOV}_{\mathrm{x}}, \mathrm{FOV}_{\mathrm{y}}\right)$

Resolution $(\Delta x, \Delta y)$

Number of data points collected $\left(N_{\mathrm{x}}, N_{\mathrm{y}}\right)$

Slice thickness $(\Delta z)$

Number of slices

Slice gap

Number of acquisitions $\left(N_{\text {acq }}\right)$

Swap read and phase encoding

Saturation pulses

Fat suppression

Scan time
Supine

Fast spin echo

Coronal oblique

FSE-XLC

Parallel to the long axis of the spine of the scapula
$12.4 \mathrm{msec}$
$17.86 \mathrm{KHz}$
2
$600 \mathrm{msec}$
$90^{\circ}$
$140 \mathrm{~mm}, 140 \mathrm{~mm}$
$0.55 \mathrm{~mm}, 0.73 \mathrm{~mm}$
256, 192
$3 \mathrm{~mm}$
24
$1 \mathrm{~mm}$
2
Yes
Inferior
Yes
$5 \mathrm{~min}, 53 \mathrm{sec}$

Table A22.2.6 Clinical Parameters for Sequence 5: Coronal Oblique

$T_{2}$-Weighted

Patient position

Scan type

Imaging plane (orientation)

Pulse sequence database (PSD)

Central slice or volume center

Echo time $\left(T_{\mathrm{E}}\right)$

Echo train length (ETL)

Repeat time $\left(T_{\mathrm{R}}\right)$

Flip angle (FA)

Fields of view $\left(\mathrm{FOV}_{\mathrm{x}}, \mathrm{FOV}_{\mathrm{y}}\right)$

Resolution $(\Delta x, \Delta y)$

Number of data points collected $\left(N_{\mathrm{x}}, N_{\mathrm{y}}\right)$

Slice thickness $(\Delta z)$

Number of slices

Slice gap

Number of acquisitions $\left(N_{\text {acq }}\right)$

Swap read and phase encoding

Saturation pulses

Fat suppression

Scan time
Supine

Fast spin echo

Coronal

FSE-XL

Parallel to the long axis of the spine of the scapula

$102 \mathrm{msec}$

9

$3375 \mathrm{msec}$

$90^{\circ}$

$140 \mathrm{~mm}, 140 \mathrm{~mm}$

$0.55 \mathrm{~mm}, 0.73 \mathrm{~mm}$

256, 192

$3 \mathrm{~mm}$

24

$1 \mathrm{~mm}$

2

Yes

Inferior

Yes

$2 \mathrm{~min}, 35 \mathrm{sec}$ 
The grid lines should begin $2 \mathrm{~cm}$ medial to the glenoid and extend beyond the lateral margin of the humeral head. Run sequence 3 following the parameters in Table A22.2.4.

19. Let the patient know that you will begin and scan.

\section{Sequence 4: Coronal oblique fat-suppressed fast spin echo (FSE) $T_{1}$-weighted}

20. The coronal oblique images are also planned from the transverse images in one of two ways. One can choose a high transverse section at the level of the supraspinatus tendon allowing direct alignment of the localizer with the tendon. The localizer should extend to the soft tissue margins both anteriorly and posteriorly. In this case,

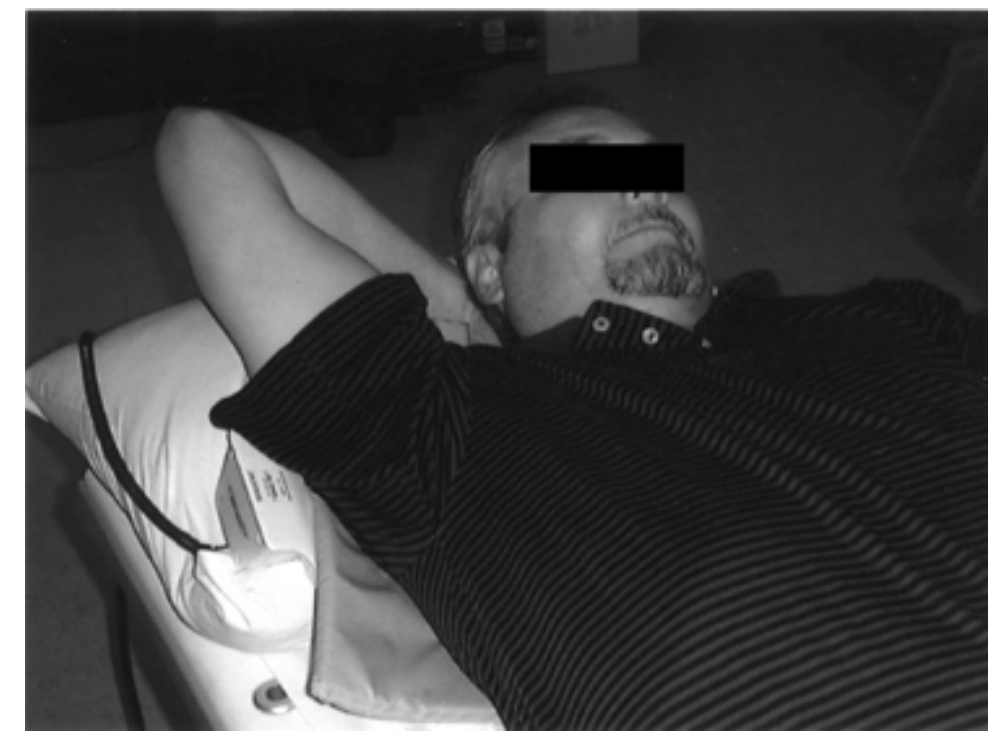

Figure A22.2.6 ABER position with coil placement. See the abducted arm and the coil behind the axilla.

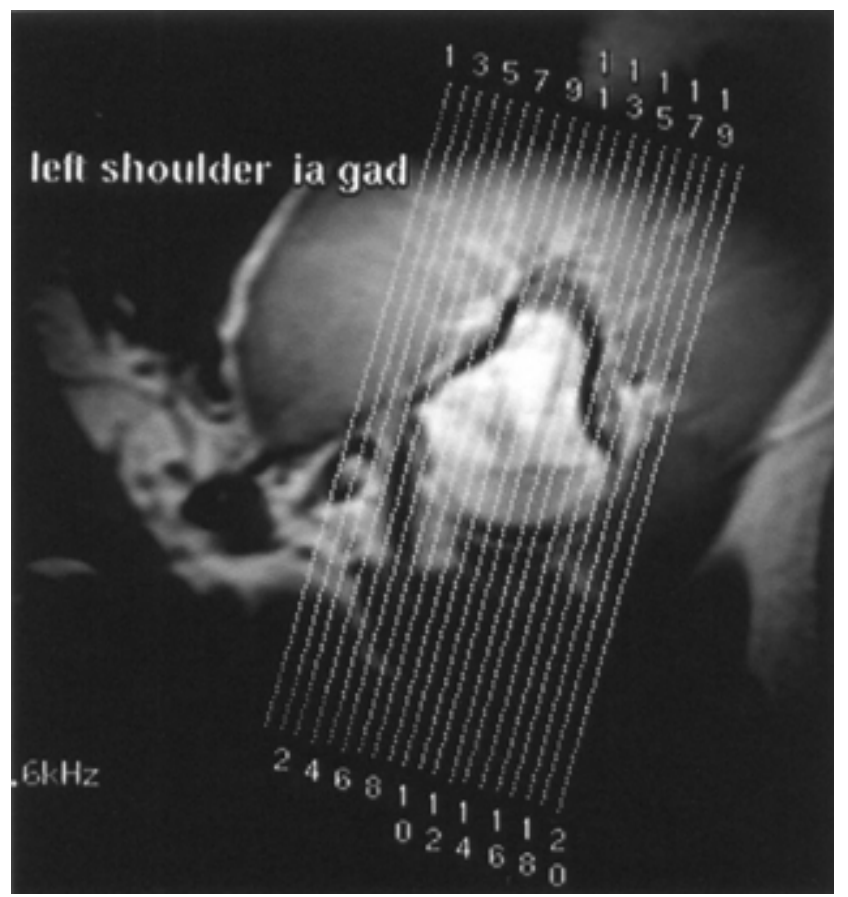

Figure A22.2.7 Localizer images parallel to the long axis of the humerus. 
the localizer lines are placed along the long axis of the visualized scapular spine, and perpendicular to the glenoid articular surface (see Fig. A22.2.4). The lines should extend $2 \mathrm{~cm}$ anterior and posterior to the humeral head. The latter method underestimates the degree of obliquity needed to section the supraspinatus tendon along its true long axis (see Fig. A22.2.5). Run sequence 4 according to the parameters in Table 22.2.5.

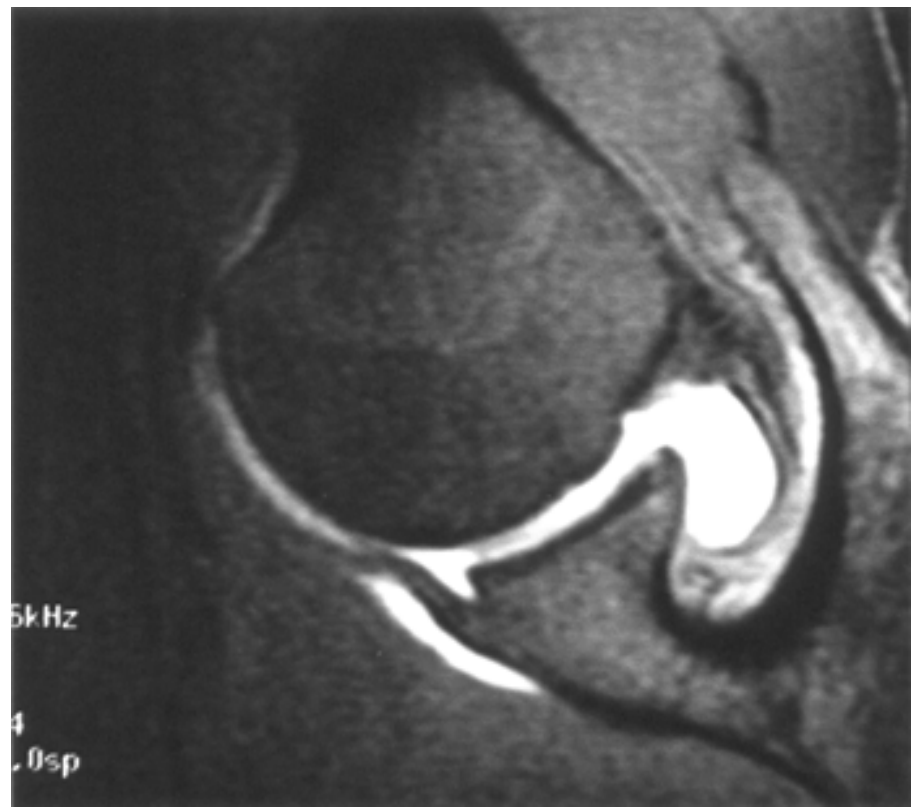

Figure A22.2.8 Example of the transverse oblique in the ABER position. This picture also shows a Perthes lesion (designation for an avulsion of the anteroinferior portion of the glenoid labrum without displacement and with stripping of the periosteal membrane).

Table A22.2.7 Clinical Parameters for Sequence 6: Spoiled Gradient-Recalled (SPGR) Coronal Localizer for ABER Position (Pilot Scan)

Patient position

Scan type

Imaging plane (orientation)

Central slice or volume center

Echo time $\left(T_{\mathrm{E}}\right)$

Repeat time $\left(T_{\mathrm{R}}\right)$

Flip angle (FA)

Fields of view $\left(\mathrm{FOV}_{\mathrm{x}}, \mathrm{FOV}_{\mathrm{y}}\right)$

Resolution $(\Delta x, \Delta y)$

Number of data points collected $\left(N_{\mathrm{x}}, N_{\mathrm{y}}\right)$

Slice thickness $(\Delta z)$

Number of slices

Slice gap

Number of acquisitions $\left(N_{\text {acq }}\right)$

Swap read and phase encoding

Saturation pulses

Scan time
Supine

2-D spoiled gradient-recalled

Coronal

Center of anatomy

$4.2 \mathrm{msec}$

$150 \mathrm{msec}$

$60^{\circ}$

$360 \mathrm{~mm}, 360 \mathrm{~mm}$

$1.41 \mathrm{~mm}, 2.82 \mathrm{~mm}$

256, 128

$7 \mathrm{~mm}$

13

$2 \mathrm{~mm}$

2

No

None

$40 \mathrm{sec}$ 
Sequence 5: Coronal oblique fast spin echo (FSE) $T_{2}$-weighted

21. The same localizer is used for this sequence 5, run this one according to the parameters in Table A22.2.6.

\section{Change position and coil}

22. Move the scanning table out of the magnet and remove the phased array shoulder coil. Ask the patient to place his or her arm in an abducted and externally rotated position (ABER), i.e., hand behind the head or neck while remaining supine (see Fig. A22.2.6). This can be an uncomfortable position for patients with shoulder pain. The importance of the sequence should be emphasized to the patient to encourage full cooperation with this potentially difficult position. A general purpose surface coil is placed between the shoulder and the scanning table. The scanning table is centered localizing the region of the shoulder and the patient is returned to the magnet.

\section{Sequence 6: Spoiled gradient-recalled (SPGR) coronal localizer for ABER position}

23. Image the upper extremity, axilla and thorax with this large field of view localizer. From this, the transverse oblique images are planned. An image should be chosen that shows the humeral shaft. Place the localizer lines along the longitudinal axis of the humeral shaft, with the center point over the glenohumeral joint (see Fig. A22.2.7). This results in an transverse oblique set of images (see Fig. A22.2.8). Run sequence 6 according to the parameters in Table A22.2.7.

\section{Sequence 7: ABER fat-suppressed fast spin echo $T_{1}$-weighted}

24. Check to be sure the parameters in Table A22.2.8 have been entered correctly. Assure the patient that this is the last sequence to be performed and again request full cooperation with respect to lack of motion. Run this sequence following the parameters in Table A22.2.8.

Table A22.2.8 Clinical Parameters for Sequence 7: ABER-Transverse Fat-Suppressed Fast Spin Echo (FSE) $T_{1}$-Weighted

\begin{tabular}{ll}
\hline Patient position & Supine \\
Scan type & Fast spin echo \\
Imaging plane (orientation) & Transverse oblique \\
Pulse sequence database (PSD) & FSE-XLC \\
Central slice or volume center & Center to the glenoid \\
Echo time $\left(T_{\mathrm{E}}\right)$ & $12.2 \mathrm{msec}$ \\
Receiver bandwidth $(\mathrm{RBW})$ & $17.86 \mathrm{KHz}$ \\
Echo train length $(\mathrm{ETL})$ & 2 \\
Repeat time $\left(T_{\mathrm{R}}\right)$ & $600 \mathrm{msec}$ \\
Flip angle $(\mathrm{FA})$ & $90^{\circ}$ \\
Fields of view (FOV, FOV & \\
Resolution $(\Delta x, \Delta y)$ & $160 \mathrm{~mm}, 160 \mathrm{~mm}$ \\
Number of data points collected $\left(N_{\mathrm{x}}, N_{\mathrm{y}}\right)$ & $0.63 \mathrm{~mm}, 0.83 \mathrm{~mm}$ \\
Slice thickness $(\Delta z)$ & 256,192 \\
Number of slices & $3 \mathrm{~mm}$ \\
Slice gap & 19 \\
Number of acquisitions $\left(N_{\mathrm{acq}}\right)$ & $1 \mathrm{~mm}$ \\
Swap read and phase encoding & 2 \\
Saturation pulses & No \\
Fat suppression & Inferior \\
Scan time & Yes \\
\hline
\end{tabular}




\section{COMMENTARY}

\section{Background Information}

The shoulder is clearly one of the most biomechanically complex articulations in the body. As previously mentioned, it has the greatest range of motion of any peripheral joint, but is the most frequently dislocated. The body has countered by providing an intricate, layered stabilizing system comprised of the osseous infrastructure, the glenoid labrum, the glenohumeral ligaments (capsular thickenings), the joint capsule, the rotator cuff, and supporting muscles. Despite the excellent soft tissue contrast afforded by MR imaging, identifying the redundant, delicate supporting layers of the glenohumeral joint can prove challenging. The distention of the joint capsule with contrast material in conjunction with specialized arm positioning offers a welcome advantage for identification of the structures associated with shoulder stability (Tirman et al., 1994).

Common indications for MR arthrography of the shoulder include any history of instability (especially previous dislocation), a suggestion of undersurface tear of the rotator cuff, or rotator cuff interval abnormality (Flanagan et al., 1990; Chung et al., in press).

Other methods for evaluation of glenohumeral joint instability have included conventional MR imaging (Gusmer et al., 1996), and indirect MR arthrography (Vahlensieck et al., 1996). Though the optimal means of evaluation of this entity remains controversial, the detailed anatomic information offered by well performed MR arthrography cannot be denied.

\section{Critical Parameters and Troubleshooting}

Clearly, anytime an invasive procedure is involved, great attention to technique must be provided. It is desirable for the injection to be entirely intraarticular. Though the shoulder is an accessible articulation, errors with contrast administration can occur and familiarity of the normal appearance of a distended joint may salvage a study. Care should be taken to avoid the introduction of air into the joint. This may result in susceptibility artifact. In addition, background work has been performed to optimize the millimolar concentration of gadolinium solution used for MR arthrography (Hajek et al., 1990). If undiluted gadolinium is placed in the joint, the result is complete loss of signal, i.e., a black appearance in the surrounding joint MR Arthrography of space.
Though fat-suppressed imaging techniques further augment image contrast, inhomogeneous suppression can prove problematic.

STIR (short tau inversion recovery) imaging is not an appropriate means of fat suppression in this setting because $T_{1}$ shortening by gadolinium-containing contrast results in decreased image contrast.

\section{Anticipated Results}

Glenohumeral joint instability is a complicated entity paralleling the complex structure and function of the articulation. Though the optimal means of imaging for evaluation of instability remains a controversial subject, the goal is accurate identification and characterization of abnormalities of the stabilizing structures of the shoulder.

\section{Acknowledgments}

The authors would like to thank R.T. Dexter Walpole for reviewing this material and providing helpful comments.

\section{Editor's Note}

This unit has been written by the authors clearly for high field scanners with fat saturation sequences. For those users who do not have fat saturation capability, such as on mid- or low-field scanners, a reasonable alternative protocol might be: (1) oblique coronal proton density weighted and $T_{2}$-weighted fast/turbo spin echo images; (2) oblique sagittal proton density weighted and $T_{2}$-weighted fast/turbo spin echo images; (3) transverse $T_{2}{ }^{*}$ gradient echo images; and (4) transverse STIR (short tau inversion recovery) images. In the future, the authors expect to have a separate unit discussing low field protocols.

Also, for a given field strength, the field of view should be adjusted to the size of the patient, within the constraints of the scanner. On a high field scanner, 12 to $14 \mathrm{~cm}$ or smaller fields of view are easily achievable and can cover the entire shoulder in an average sized patient to give the best resolution. The field of view should be adjusted down for smaller patients and up for larger patients, as necessary. On mid- and low-field scanners, these smaller fields of view may not be achievable in reasonable scan times and with sufficient signal to noise. In that case, the smallest field of view should be used (sacrificing some resolution as needed) to maintain sufficient signal to noise and the images then magnified (zoomed). 


\section{Literature Cited}

Chandnani, V.P., Yeager, T.D., DeBernardino, T., Christensen, K., Gagliardi, J.A., Heitz, D.R., and Hansen, M.F. 1993. Glenoid labral tears: Prospective evaluation with MRI imaging. MR arthrography, and CT arthrography. Am. J. Roentgenol. 161:1229-1235.

Chung, C.B., Dwek, J.D., Cho, G.J., Lektrakul, N., Trudell, D., and Resnick, D. Rotator cuff interval: Evaluation with MR imaging and MR Arthrography of the shoulder in 32 cadavers. $J$. Comput. Assisted Tomogr. In press.

Flannigan, B., Kursunoglu-Brahme, S., Snyder, S., Karzel, R., Del Pizzo, W., and Resnick, D. 1990. MR arthrography of the shoulder: Comparison with conventional MR imaging. Am. J. Roentgenol. 155:829-832.

Gusmer, P.B., Potter, H.G., Schatz, J.A., Wickiewicz, T.L., Altchek, D.W., O'Brien, S.J., and Warren, R.F. 1996. Labral injuries: Accuracy of detection with unenhanced MR imaging of the shoulder. Radiology 200:519-524.

Hajek, P.C., Sartoris, D.J., Gylys-Morin, V., Haghighi, P., Engel, A., Kramer, F., Neumann, C.H., and Resnick, D. 1990. The effect of intraarticular gadolinium-DTPA on synovial membrane and cartilage. Investig. Radiol. 25:179183.

Hajek, P.C., Sartoris, D.J., Neumann, C.H., and Resnick, D. 1987. Potential contrast agents for
MR arthrography: In vitro evaluation and practical observations. Am. J. Roentgenol. 149:97104.

Shellock, F.G. 1996. Pocket Guide to MR Procedures and Metallic Objects. Lippincott-Raven, Philadelphia.

Tirman, P.F., Bost, F.W., Steinbach, L.S., Mall, J.C., Peterfy, C.G., Sampson, T.G., Sheehan, W.E., Forbes, J.R., and Genant, H.K. 1994. MR arthrographic depiction of tears of the rotator cuff: Benefit of abduction and external rotation of the arm. Radiology 192:851-856.

Vahlensieck, M., Peterfy, C.G., Wischer, T., Sommer, T., Lang, P., Schlippert, U., Genant, H.K., and Schild, H.H. 1996. Indirect MR arthrography: Optimization and clinical applications. $R a$ diology 200:249-254.

Zanetti, M. and Hodler, J. 1997. Contrast media in MR arthrography of the glenohumeral joint: Intra-articular gadopentetate vs saline: Preliminary results. Eur. Radiol. 7:498-502.

Contributed by Christine B. Chung, Leopoldo M. Gigena, and Donald Resnick Veterans Affairs San Diego Healthcare System

University of California San Diego

La Jolla, California 